\title{
Extensions of adaptive slope-seeking for active flow control
}

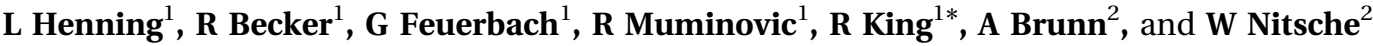 \\ ${ }^{1}$ Department of Measurement and Control, Technische Universität Berlin, Berlin, Germany \\ ${ }^{2}$ Department of Aeronautics and Astronautics, Technische Universität Berlin, Berlin, Germany
}

The manuscript was received on 1 October 2007 and was accepted after revision for publication on 7 February 2008.

DOI: 10.1243/09596518JSCE490

\begin{abstract}
To speed up gradient estimation in a slope-seeking controller two different modifications are proposed in this study. In a first approach, the gradient estimation is based on a locally identified black-box model. A further improvement is obtained by applying an extended Kalman filter to estimate the local gradient of an input-output map. Moreover, a simple method is outlined to adapt the search radius in the classical extremum- and slopeseeking approach to reduce the perturbations near the optimal state. To show the versatility of the slope-seeking controller for flow control applications two different wind tunnel experiments are considered, namely with a two-dimensional bluff body and a generic threedimensional car model (Ahmed body).
\end{abstract}

Keywords: active flow control, extremum-seeking control, slope-seeking control, Kalman filter, least square, Ahmed body, bluff body

\section{INTRODUCTION}

The constantly rising requirements in the operation of turbo-machines, burners, or transport vehicles with respect to energy consumption, pollution, noise emission, and other performance criteria have led to a growing interest in improvements of flow control. On the one hand, various passive means for flow control, such as spoilers, vortex generators or disruptors, etc., are well-investigated and are used in numerous applications, (cf. references [1] to [4]). On the other hand, active flow control can further improve the performance and stretch the dynamic range of operation by exploiting additional degrees of freedom. The majority of the work done so far in the field of active flow control concentrates on openloop concepts $[\mathbf{5}, \mathbf{6}]$. However, as generally known, open-loop control suffers from the effects of uncertainties. To synthesise closed-loop flow controllers instead, various routes can be taken. The synthesis may be based on the evolution equations of viscous fluid flow, i.e. the Navier-Stokes and the continuity

*Corresponding author: Department of Plant and Process Technology, Technische Universität Berlin, Hardenbergstrasse 36a, Berlin 10623, Germany. email: Rudibert.King@TU-Berlin.de equations (e.g. references [7] and [8]). As the requirement of a real-time capability has to be kept in mind, this computationally intensive approach will not be found in experiments in the near future. Three other promising approaches remain, as follows.

1. Potentially good results are obtained by nonlinear controllers exploiting low-dimensional models that describe the non-linear physics, such as Galerkin or vortex models [9-14]. These models are still restricted to rather simple flow configurations. Experimental validations are rare.

2. Based on an off-line identified family of linear or non-linear black-box models a fast and cheap controller synthesis employing robust methods such as $H_{\infty}$ or QFT design is possible (see, for example, references [15] to [18]).

3. Finally, adaptive methods, either model-based [19] or without explicit models, can be used.

All of the last three approaches have been investigated by the authors and have been tested in numerous experimental studies. This contribution focuses on the most simple approach employing model-free adaptive methods, i.e. extremum-seeking control. The aim of the paper is twofold. 
1. By considering different new flow configurations, namely a two- and a three-dimensional bluff body, the versatility and ease of application of this last approach is demonstrated. Other applications of extremum-seeking control of the authors for noise reduction in turbo-machines, flow separation control in diffusors, and mitigation of thermoacoustic instabilities in burners can be found in references [20] to [22].

2. Extensions are proposed to tackle the major disadvantage of extremum-seeking controllers, which is the very low closed-loop bandwidth, and to adapt a search radius.

However, it should be noted that the authors believe that all methods proposed above will be needed - in some cases simultaneously - depending on the closed-loop specifications.

This paper has a clear focus on experimental applications of closed-loop flow control as similar studies are still very scarce. Moreover, as stability proofs of classical extremum-seeking controllers are already known $[\mathbf{2 3}, \mathbf{2 4}]$ and more theoretical studies are hampered due to the very nature of the control algorithms, this contribution is more of an explanatory style. The proposed extensions of the controller are empirical in nature, and detailed closed-loop stability proofs are not presented.

For both configurations considered in the present work, extensive passive and open-loop active flow control studies can be found. Disregarding passive approaches, an active flow control application for a two-dimensional bluff body with spanwise distributed forcing at the trailing edges of the bluff body is described in reference [25]. A beneficial effect of active base bleeding for drag reduction is observed in references [26] and [27]. The closed-loop flow control of two-dimensional bluff bodies mainly concentrates on a well-established benchmark problem: the flow around a circular cylinder. In reference [14] a simple low-dimensional model is used in the controller. In reference [28] the synthesis of various non-linear controllers for this flow is reported. The reduction in drag of another bluff body is achieved in reference [29] by application of a rotating cylinder located at the separation line. An adaptive extremum-seeking controller has been used to find the optimal speed of rotation of the cylinder for maximum drag reduction in this application. In reference [30] the control is considered of the wake behind a so-called D-shaped bluff body with a robust controller based on a QFT design.

In the case of three-dimensional bluff bodies the number of experimental studies on open- and closed-loop control is significantly lower (cf. references [31] and [32]). The most prominent threedimensional bluff bodies are cars and trucks.

The paper is organized as follows. The extremumand slope-seeking control is reviewed in section 2. To improve the closed-loop bandwidth of these wellknown approaches and to adapt the search radius some new extensions are also proposed in this section. In section 3 experimental results are given for two different applications. Finally, the paper closes with a conclusion and an outlook in section 4 .

\section{EXTREMUM-SEEKING CONTROLLER}

The extremum- and slope-seeking feedback controllers are adaptive gradient-based, model-independent feedback schemes that search for optimal actuation parameters $[\mathbf{2 1}, \mathbf{2 9}, \mathbf{3 3}, \mathbf{3 4}]$. Whereas the original idea $[\mathbf{3 5}]$ is rather old, the interest in extremum-seeking control was boosted more than 40 years later with the stability proofs found in references [23] and [24]. An extremum-seeking control can be used to find areas around distinct minima or maxima in the steady state map of a plant. As many flow applications are rather characterized by a plateau-type map, slope-seeking is better suited. Here, the system is driven to a preset reference slope which is representative for a value just below the plateau. Two different configurations are used in this study, a single-input single-output (SISO) and a multiple-input singleoutput (MISO) slope-seeking controller. Extensions to the truly multiple-input case can be found in reference [20]. As the extremum-seeking controller forms the basis for the slope-seeking variant, the former is reviewed first.

\subsection{SISO extremum-seeking control}

The extremum-seeking control is a model-free method for the control of non-linear plants characterized by an output extremum in the steady state $[23,24,35]$ and for linear or non-linear plants for which the output is defined as the norm of the difference between a reference value $r(t)$ and the plant output $y(t)$, e.g. $[r(t)-y(t)]^{2}$ in an SISO setting. As every minimization problem can easily be transformed into a maximization problem, all explanations are given for the latter case. Figure 1 shows the structure of the basic SISO extremumseeking control loop. Here, the process is described by both its steady state map $y_{s}=f\left(u_{s}\right)$ and its dynamical model for ease of further discussion. 


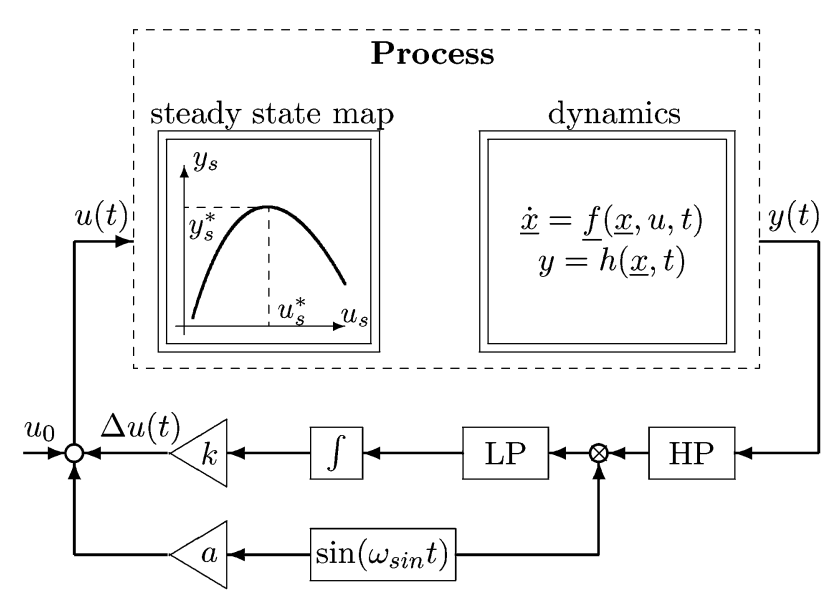

Fig. 1 Block diagram of closed-loop extremum-seeking. The HP and LP represent high- and lowpass filters respectively

The idea of this gradient-based method is an on-line optimization of the average value, $u_{s}$, of the control input $u(t)$ such that the average of the output equals the maximum steady state value, $y_{s}=y_{s}^{*}$. With extremum-seeking control this can be accomplished without knowing the steady state input-output map $y_{s}=f\left(u_{s}\right)$. The controller works as follows. Assume that the initial control input $u_{0}$ (see Fig. 1), which is calculated by some higher level control hierarchy or set to zero, is superimposed with a sinusoidal signal $a \sin \left(\omega_{\sin } t\right)$, which has a small amplitude $a$

$$
u(t)=u_{0}+a \sin \left(\omega_{\sin } t\right)[+\Delta u(t)]
$$

If the period of this harmonic perturbation is larger than the largest time constant of the process, the output of the process will also be approximately sinusoidal, centred initially around $y_{s, 0}=f\left(u_{0}\right)$. Likewise, the amplitude will be approximately $a f^{\prime}$. Hence

$$
y(t) \approx y_{s}+a f^{\prime} \sin \left(\omega_{\sin } t\right)
$$

This output perturbation is analysed in order to detect the slope (gradient) of the input-output map, which is used for gradient-based optimization. To do this, the mean value $y_{s}$ is removed by a first-order high-pass (HP) filter

$$
G_{\mathrm{HP}}(\mathbf{j} \omega)=\frac{\mathbf{j} \omega}{\mathbf{j} \omega+\omega_{\mathrm{HP}}}
$$

with the cut-off frequency $\omega_{\mathrm{HP}}$, or by a first-order band-pass (BP) filter

$$
G_{\mathrm{BP}}(\mathbf{j} \omega)=\frac{\mathbf{j} \omega \omega_{2}}{\left(\mathbf{j} \omega+\omega_{1}\right)\left(\mathbf{j} \omega+\omega_{2}\right)}
$$

with the lower cut-off frequency $\omega_{1}$ and the upper cut-off frequency $\omega_{2}$. By application of an HP filter the output of the filter reads

$$
y_{\mathrm{HP}}(t) \approx\left|G_{\mathrm{HP}}\left(\mathrm{j} \omega_{\sin }\right)\right| a f^{\prime} \sin \left(\omega_{\sin } t+\varphi_{\mathrm{HP}}\right)
$$

The product $y_{P}(t)$ of this filtered output and the zeromean sine signal $\sin \left(\omega_{\sin } t\right)$ indicates the slope of the unknown map $y_{s}=f\left(u_{s}\right)$

$$
\begin{aligned}
y_{P}(t) & =\sin \left(\omega_{\sin } t\right)\left[\left|G_{\mathrm{HP}}\left(\mathrm{j} \omega_{\sin }\right)\right| a f^{\prime} \sin \left(\omega_{\sin } t+\varphi_{\mathrm{HP}}\right)\right] \\
& =\left|G_{\mathrm{HP}}\left(\mathrm{j} \omega_{\sin }\right)\right| a f^{\prime}\left[\sin ^{2}\left(\omega_{\sin } t\right) \cos \left(\varphi_{\mathrm{HP}}\right)\right. \\
& \left.+\sin \left(\omega_{\sin } t\right) \cos \left(\omega_{\sin } t\right) \sin \left(\varphi_{\mathrm{HP}}\right)\right]
\end{aligned}
$$

This product leads to a non-zero mean signal obtained with a low-pass (LP) filter (see Fig. 1), as long as the maximum is not obtained. If the plant is initially to the left of the maximum, the input and output perturbations are in-phase; i.e. the product will be positive. An antiphase relation that gives a negative product is an indication that the plant is on the right of the maximum. To see this, approximate the output $y_{\mathrm{LP}}(t)$ of the LP filter by an average calculated for one period $T=2 \pi / \omega_{\text {sin }}$, i.e.

$$
\begin{aligned}
y_{\mathrm{LP}}(t) & \approx \frac{1}{T} \int_{0}^{T} y_{P}(t) \mathrm{d} t \\
& =\frac{1}{T}\left|G_{\mathrm{HP}}\left(\mathrm{j} \omega_{\mathrm{sin}}\right)\right| a f^{\prime} \frac{T}{2} \cos \left(\varphi_{\mathrm{HP}}\right) \\
& =\frac{a f^{\prime}}{2} \Re e\left\{G_{\mathrm{HP}}\left(\mathrm{j} \omega_{\sin }\right)\right\}
\end{aligned}
$$

With this information an additional term $\Delta u(t)$, added to $u_{0}+a \sin \left(\omega_{\sin } t\right)$ (see equation (1) and Fig. 1), is calculated by time integration and multiplication by $k$. As long as the output of the LP filter is positive, i.e. the system is on the left side of the maximum, a steadily increasing control input $u$ is obtained. For a negative output of the LP filter, the opposite is true. The adaptation of $u$ converges to $u=u^{*}$.

The extremum-seeking scheme is an adaptive closed-loop type of control. It guarantees closedloop stability if designed properly (see references [23] and [24] for details). The choice of certain design parameters determines the speed of convergence. The cut-off frequency of the HP filter needs to be lower than the frequency $\omega_{\sin }$ of the perturbation 
signal. Thus, ideally, the overall feedback system has fast, medium, and slow time scales corresponding to the plant dynamics, the periodic perturbation, and the filter respectively. For the results given below, however, the HP filter is replaced by a BP filter. With $\omega_{\mathrm{BP}}=\omega_{1}=\omega_{2}=\omega_{\text {sin }}$ the output perturbation passes the BP filter, high-frequency disturbances can be damped, and a phase shift can be avoided. The LP filter is not necessary, but it is helpful in filtering out the perturbation after the multiplier. Therefore, the cut-off frequency of the LP filter should be chosen for $\omega_{\mathrm{LP}} \leqslant \omega_{\mathrm{sin}}$. In addition, the adaption gain $k$ needs to be small. If the plant behaviour varies due to uncertainties, the time scale of the perturbation signal has to be slower than the slowest possible plant dynamic. The main advantage of this extremum-seeking control is that no plant model is needed for controller synthesis. However, the control suffers from both the permanent harmonic input and output perturbations and the relative slow dynamic behaviour. Therefore, some extensions will be proposed later.

\subsection{Slope-seeking as a generalized design}

In flow control, situations are encountered in which the static input-output map does not show a distinct maximum. Instead, it is characterized by a plateautype behaviour, as illustrated in Fig. 2. A detached flow over an aircraft wing, to give an example, can be influenced by active flow control such that it reattaches again. At some point, however, the flow is completely attached. A further increase of the control input will not result in a further increase nor in a decrease of the lift. The extremum-seeking control described above will work correctly in this

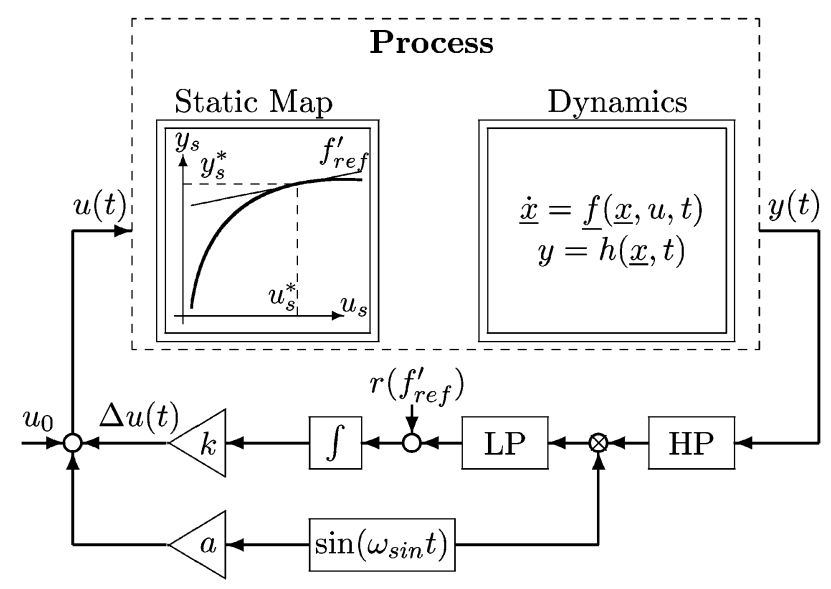

Fig. 2 Block diagram of closed-loop slope-seeking situation if it starts left of the plateau. However, when the control input is larger than the smallest value necessary to be on top of the plateau, it will not be minimized. Such a waste of control energy can be observed likewise when the system's behaviour due to a change in operating conditions - will change. In such a case, the plateau-type map might be shifted to the left. The smallest value necessary to be on top of the plateau found for the last operating condition now lies somewhere on the plateau without any gradient information for the controller. To tackle such systems, slope-seeking is considered next.

Slope-seeking is an extension of the extremumseeking scheme (for details see reference [24]). It drives the plant output to a value that corresponds to a reference slope of the steady state input-output map

$$
f_{\text {ref }}^{\prime}=\left.\frac{\partial y_{\mathrm{s}}}{\partial u_{\mathrm{s}}}\right|_{\mathrm{ref}}
$$

Therefore, according to equation (7), a negative reference value

$$
r\left(f_{\text {ref }}^{\prime}\right)=-\frac{a f_{\text {ref }}^{\prime}}{2} \Re e\left\{G_{\mathrm{HP}}\left(\mathrm{j} \omega_{\text {sin }}\right)\right\}
$$

as a function of $f_{\text {ref }}^{\prime}$ is added to the actually detected slope (see Fig. 2). Thus, the apparent extremum is shifted. Since extremum-seeking is a special case of slope-seeking, when the reference slope is zero, designing the filters, the integrator gain, and the sinusoidal perturbation are the same.

\subsection{Adaptation of the search radius}

A controller operating in the desired optimal point is wasting actuation energy because of the permanent perturbation of the control input. For a maximum with a rapidly growing decline in the vicinity [20], the performance deteriorates because, due to the harmonic perturbation, the process circles far downhill around the extremum. Therefore, an adaptation of the amplitude would be beneficial. At the optimal point a smaller amplitude is desired, whereas a high amplitude far away from the optimal point speeds up adaptation of $u$. With aggressive methods, i.e. methods that are too fast, that are used to adjust $a$ it is impossible, however, to avoid interference with the $u$ adaptation.

A proposed extension is shown in Fig. 3. The block diagram of the controller is extended by a loop in 


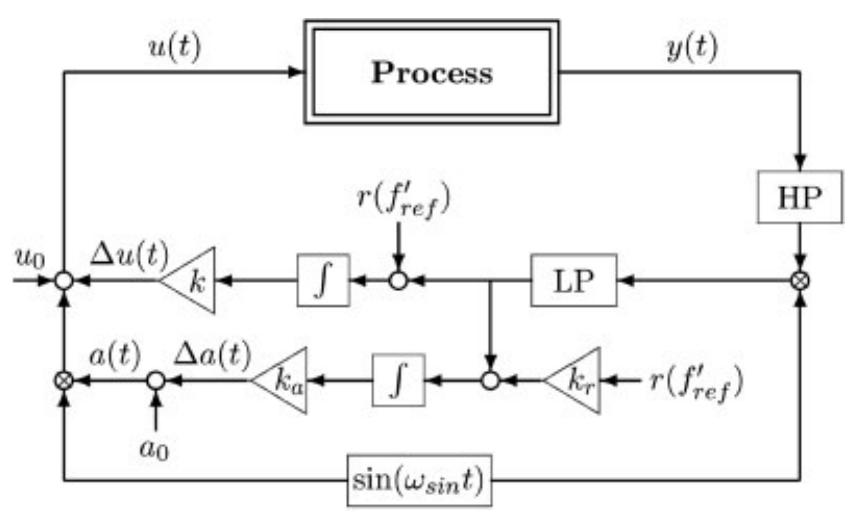

Fig. 3 Block diagram of closed-loop slope-seeking with an extension for the adaptation of the perturbation amplitude

order to adjust the perturbation amplitude $a(t)$. For high local gradients, a multiplied reference value $r\left(f_{\text {ref }}^{\prime}\right)$ added to the output of the LP filter yields a positive input into the new integrator. Accordingly, the input of the integrator is negative for small local gradients of the steady state map, as also seen in equation (7). Hence, the amplitude adjustment $\Delta a(t)$ is calculated by time integration and multiplication by the gain $k_{a}$ similar to the determination of $\Delta u(t)$.

The adaptation of $u(t)$ and $a(t)$ have to be dynamically decoupled. The adaptation of the perturbation amplitude has to be slower than the adaptation of $u(t)$, which can be achieved by choosing the gain $k_{a}$ smaller than $k$. Hence, similar arguments follow concerning stability as for the extremum-seeking controller itself.

\subsection{Least squares estimation}

In order to improve the gradient estimation it is proposed to modify the slope-seeking controller. The filters and the sine signal for perturbation are replaced by a system identification approach to determine the local gradient. The basic concept is shown in Fig. 4 . In every time interval $\left[t_{k-1}, t_{k}\right]$ the control input changes stepwise from the current input $u\left(t_{k-1}\right)$ successively to $u\left(t_{k-1}\right)+a$ and then to $u\left(t_{k-1}\right)-a$. According to the classical slope-seeking algorithm, $a$ is the actuation amplitude and the length of the time interval corresponds to the period of the perturbation. At every time step $k$ an estimation update is performed, in which the two input/output data sets are used for two on-line parameter estimations (positive and negative steps). A standard least squares algorithm (for details see references [36] and [37]) is applied to estimate the parameters $\alpha_{i}$ and $\beta_{i}$ of the $m$ th-order rational transfer function

$$
G(q)=\frac{\beta_{0}+\beta_{1} q^{-1}+\beta_{2} q^{-2}+\cdots+\beta_{m} q^{-m}}{1+\alpha_{1} q^{-1}+\alpha_{2} q^{-2}+\cdots+\alpha_{m} q^{-m}}
$$

with the shift operator $q$.

Based on the estimated parameters of the transfer functions, steady state values can be estimated for the upward and the downward steps respectively

$$
\hat{y}_{s, u p}=\frac{\sum_{i=0}^{m} \beta_{i}}{1+\sum_{i=1}^{m} \alpha_{i}}\left[u\left(t_{k-1}\right)+a\right]
$$

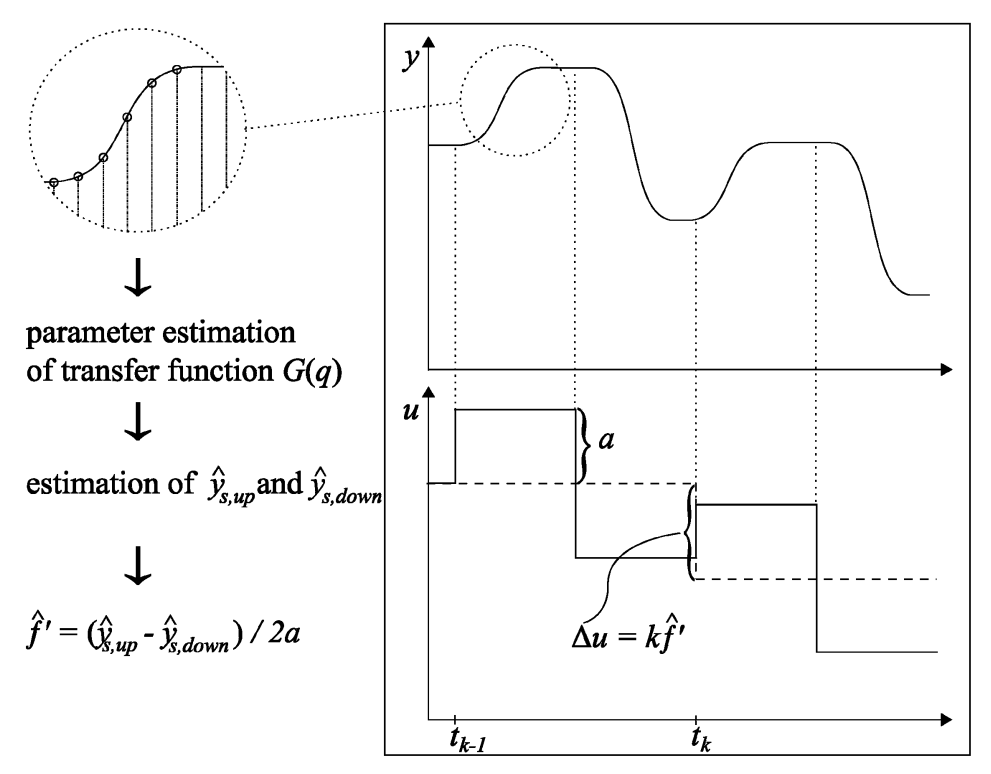

Fig. 4 Concept of the gradient estimation by application of a least squares algorithm 


$$
\hat{y}_{s, \text { down }}=\frac{\sum_{i=0}^{m} \beta_{i}}{1+\sum_{i=1}^{m} \alpha_{i}}\left[u\left(t_{k-1}\right)-a\right]
$$

The local gradient can then be approximated by

$$
\hat{f}^{\prime}=\frac{\hat{y}_{s, u p}-\hat{y}_{s, \text { down }}}{2 a}
$$

Finally, the next control input is calculated by the recursion formula

$$
u\left(t_{k}\right)=k \hat{f}^{\prime}+u\left(t_{k-1}\right)
$$

where the adaption gain $k$ determines the step size of $u$. The parameter $k$ should not be too large to guarantee the convergence of the control input.

\subsection{A Kalman filter algorithm for gradient estimation}

As in the classical extremum-seeking control it will still be assumed that the harmonic perturbation of the process is slower than the slowest time constant of the process. Hence, the static input-output map is reflected by the behaviour of the output $y(t)$. If the input perturbation $a$ is small enough, the output perturbation should be $a f^{\prime}$, as already mentioned in section 2.1. The output itself consists of an approximately constant value plus this perturbation, i.e.

$$
y(t) \approx y_{s}+a f^{\prime} \sin \left(\omega_{\sin } t\right)
$$

With equation (1)

$$
y(t) \approx \underbrace{y_{s}-f^{\prime} u_{0}-f^{\prime} \Delta u(t)}_{x_{1}}+\underbrace{f^{\prime}}_{x_{2}} u(t)=x_{1}+u(t) x_{2}
$$

The idea is to observe these two parameters $x_{1}$ and $x_{2}$. A dynamical model for the two parameters reads in discrete time

$$
\underline{\boldsymbol{x}}\left(t_{k+1}\right)=\left[\begin{array}{ll}
1 & 0 \\
0 & 1
\end{array}\right] \underline{\boldsymbol{x}}\left(t_{k}\right)+\underline{w}_{k}
$$

If equation (15) is used as a measurement equation, an observability analysis shows that $x_{1}$ and $x_{2}$ are not observable. However, when time-shifted input-output pairs $(u(t), y(t))$ and $(u(t-n \Delta t), y(t-n \Delta t))$ are considered, observability is given. With $y_{1}\left(t_{k}\right)=y\left(t_{k}\right)$ and $y_{2}\left(t_{k}\right)=y\left(t_{k-n}\right)$ the measurement equation now reads

$$
\underline{\boldsymbol{y}}\left(t_{k}\right)=\left[\begin{array}{ll}
1 & u_{1} \\
1 & u_{2}
\end{array}\right] \underline{\boldsymbol{x}}\left(t_{k}\right)+\underline{\varsigma}_{k}
$$

with $u_{1}=u\left(t_{k}\right)$ and $u_{2}=u\left(t_{k-n}\right)$. The vectors $\underline{\boldsymbol{w}}_{k}$ and $\underline{\varsigma}_{k}$ denote Gaussian white noises. An extended Kalman filter [38] can be used for real-time estimation of the states. The modification of the slopeseeking control by inclusion of this model-based sensor is shown in Fig. 5. Since the extended Kalman filter estimates the slope $\hat{f}^{\prime}=\hat{x}_{2}$, the reference slope $f_{\text {ref }}^{\prime}$ is subtracted directly from $\hat{f}^{\prime}$.

\section{APPLICATIONS}

In the following sections the utilization of the slopeseeking controller for flows with a D-shaped bluff body and a generic three-dimensional car model is described. In these two examples, extensions to accelerate the controllers and to adapt the search radius $a$ are included.

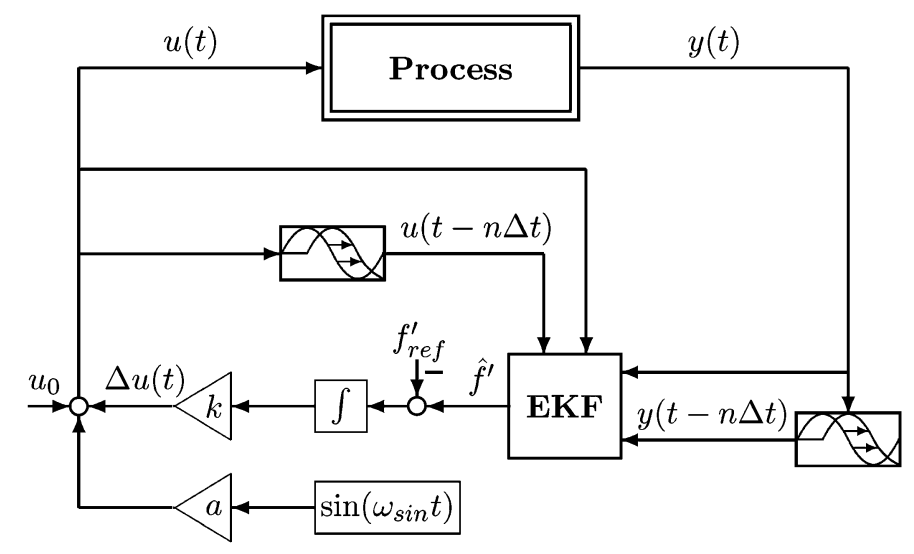

Fig. 5 Block diagram of the closed-loop slope-seeking with an extended Kalman filter (EKF) algorithm for fast real-time estimation of the local gradient of the steady state map 


\subsection{Active drag reduction of a D-shaped bluff body}

The flow separation behind bluff bodies, such as vehicles, shows complex space- and time-dependent topology that results in an increase in aerodynamic drag. A principle sketch of the investigated twodimensional D-shaped body is shown in Fig. 6 . The flow around the D-shaped body is governed by an absolute wake instability [39]. This mechanism generates a von Kármán vortex street with an alternating sequence of vortices at characteristic frequencies. The natural flow is characterized by a short dead-water region and alternating vortices in the vicinity of the base. Both are responsible for a low base pressure and thus for a high pressureinduced drag. The proposed active control strategy enforces a decoupling of the vortex formation in the shear layers and the wake by synchronizing the roll-up of upper and lower shear layers. This delays the appearance of asymmetries in the wake flow and thus mitigates the wake instability. The dead water region is enlarged and the base pressure increases [34].

A sinusoidal zero-mass actuation is applied by loudspeakers through spanwise slots located at the upper and lower trailing edges. The slot width is $s=1 \mathrm{~mm}$ and spanwise length amounts to $250 \mathrm{~mm}$. With this harmonic actuation the unstable processes of shear layer roll-up are triggered. By this means a maximum response of the flow can be generated with a minimum amount of input of energy. The actuation frequency $f_{a}$ is related to natural instability frequencies of the flow. It should not be mistaken with the perturbation frequency $\omega_{\sin }=2 \pi f_{\sin }$ of the controller.

The intensity of the actuation, which will be modified in the following, is characterized by the non-dimensional excitation momentum coefficient [6]

$$
c_{\mu}=\frac{2 s q_{\mathrm{a}}^{2}}{h u_{0}^{2}}
$$

in which $q_{a}$ is the effective velocity of the actuation.

The base pressure is monitored by $3 \times 3$ difference pressure gauges (PascaLine PCLA02X5D1) mounted in three parallel rows on the rear end at $y=\{-32,0$, $32\} \mathrm{mm}$ and $z=\{-82.5,0,82.5\} \mathrm{mm}$. The reference pressure is taken in front of the body. Four strain gauges (HBM, $350 \Omega, k$-factor 2.09) are applied to the aluminium rods supporting the model for measurement of drag. The base pressure and drag are described by the non-dimensional coefficients

$$
\begin{aligned}
& c_{p}(y, z, t)=\frac{\Delta p}{\rho u_{0}^{2} / 2} \\
& c_{\mathrm{d}}(t)=\frac{f_{x}}{\rho u_{0}^{2} h w / 2}
\end{aligned}
$$

respectively. In the present work, $\Delta p$ is the instantaneous pressure difference between a rear endmounted pressure gauge and the reference pressure, $\rho$ denotes the density, and $f_{x}$ is the drag force. Spatially and spatially-temporally averaged base pressures across the stern are marked by $c_{p b}(t)$ and $\bar{c}_{p b}$ respectively. Due to the relatively high blockage of the wind tunnel test section by the bluff body model (approximately 13 per cent), the blockage correction method proposed in reference [40] is used.

The data acquisition and the implementation of the controllers are realized on a rapid prototyping hardware (dSPACE-PPC1005 controller board). The sampling frequency is $1000 \mathrm{~Hz}$.

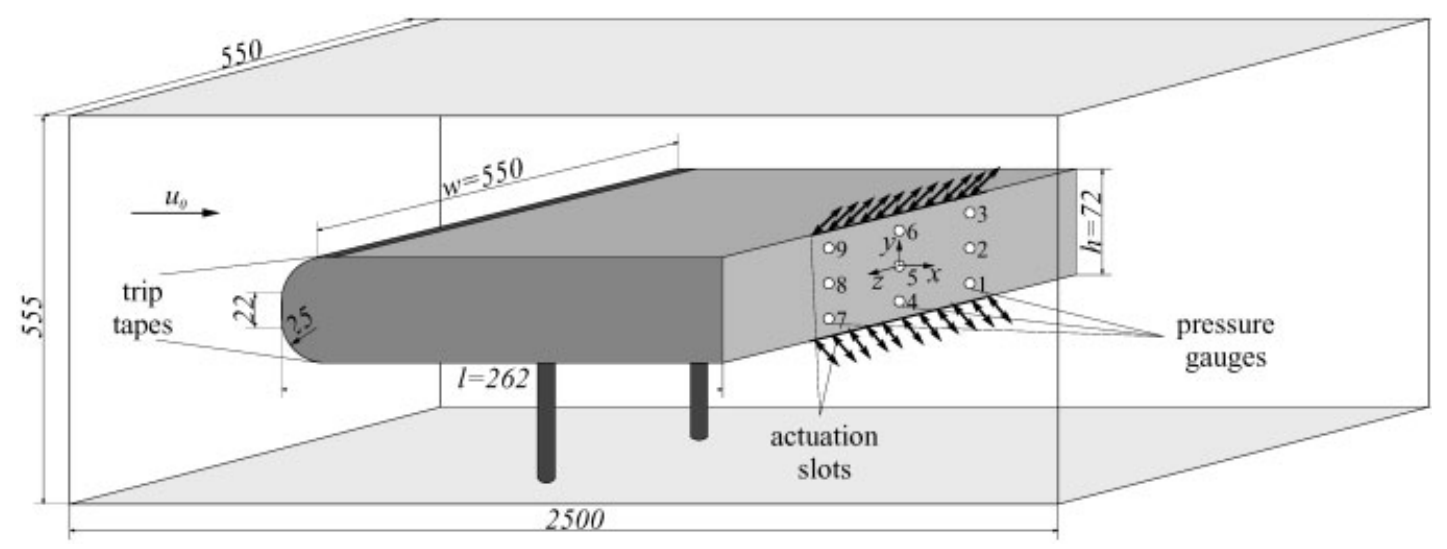

Fig. 6 Sketch of the D-shaped body and the test section. All dimensions are in mm. Trip tapes are placed $30 \mathrm{~mm}$ downstream of the nose in order to trigger boundary layer transition 
$(a)$

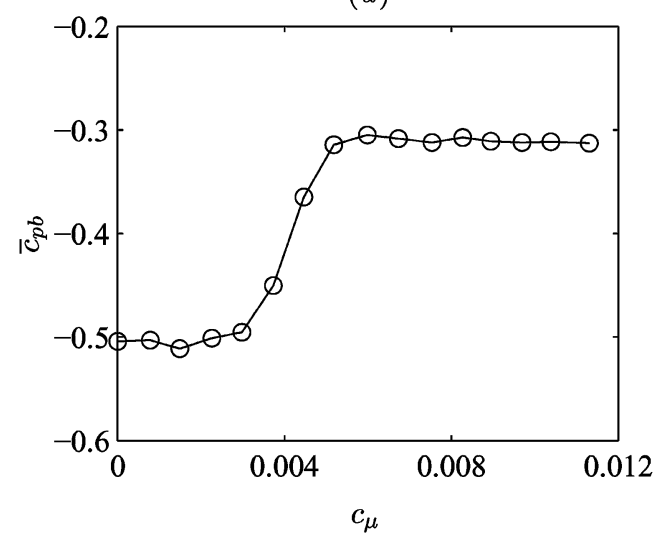

(b)
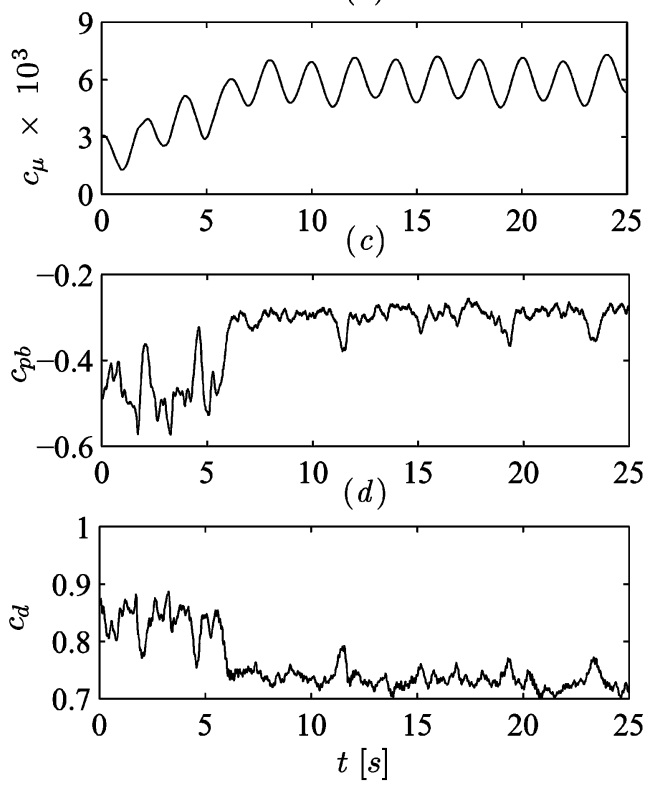

Fig. 7 Steady state map $\bar{c}_{p b}=f\left(c_{\mu}\right)$ obtained from open-loop experiments (a) and experimental implementation of slope-seeking feedback for optimal drag reduction of the D-shaped body (b-d). The controller adapts the actuation amplitude (b) until the maximum base pressure (c) has been reached and a maximum drag reduction of approximately 15 per cent can be achieved. All experiments are performed at Reynolds number 46000 with inphase forcing with a Strouhal number $S t_{a}=0.15$

\subsubsection{Classical slope-seeking control}

Figure 7(a) shows the steady state map with the time-averaged base pressure coefficient as a function of the momentum coefficient at a constant Reynolds number $R e_{h}=46000$ obtained from openloop experiments in reference [34]. The Reynolds number is calculated with the body height $h$ and free stream velocity $u_{0}$. Both actuators operate in-phase with an optimal frequency $S t_{a}=f_{a} h / u_{0}=0.15$, which was indicated as the most effective actuation parameter for synchronization of the shear layer development and for drag reduction in reference [34]. This steady state map is characterized by a plateau for $c_{\mu} \geqslant 6 \times 10^{-3}$. In order to achieve maximum base pressure with the minimum control input a slope-seeking controller with reference slope $f_{\text {ref }}^{\prime}=5$ is applied.

In the present study, the momentum coefficient is chosen as the input variable $u(t)=c_{\mu}(t)$, and the output is given by the spatially averaged base pressure coefficient $y(t)=c_{p b}(t)$. A detailed list of the controller parameters can be found in Table 1 .

The experimental data for a single operating point are shown in Figs 7(b) to (d). The controller starts at $c_{\mu}=2 \times 10^{-3}$. The sinusoidal modifications of $c_{\mu}$ are applied to obtain the local slope. According to the gradient, the actuation amplitude is raised until a state in front of the plateau is reached. This leads to a significant increase in the base pressure coefficient, as shown in Fig. 7(c), corresponding to the steady state map. A reduction of the drag coefficient by 15 per cent can be observed.

To show disturbance rejection of this controller, the Reynolds number is increased continuously from 38000 up to 70000 in Fig. 8(d). Corresponding to the steady state maps for various Reynolds numbers shown in Fig. 8(a), the optimal actuation amplitude is automatically decreased with increasing Reynolds number. The experimental results in Figs 8(b) and (c) indicate that the desired averaged base pressure $\bar{c}_{p b}=-0.3$ is maintained with the minimum control input.

Table 1 Parameters of standard slope-seeking controller in the applications

\begin{tabular}{lll}
\hline Parameter & D-shaped body & Ahmed body \\
\hline Perturbation frequency $\omega_{\text {sin }}$ & $3.14 \mathrm{rad} / \mathrm{s}$ & $3.14 \mathrm{rad} / \mathrm{s}$ \\
Perturbation amplitude $a$ & $1.3 \times 10^{-3}$ & $4 \times 10^{-4}$ \\
Reference slope $f_{\text {ref }}^{\prime}$ & 5 & 40 \\
Cut-off frequency BP $\omega_{\mathrm{BP}}$ & $3.14 \mathrm{rad} / \mathrm{s}$ & $3.14 \mathrm{rad} / \mathrm{s}$ \\
Cut-off frequency LP $\omega_{\mathrm{LP}}$ & $3.14 \mathrm{rad} / \mathrm{s}$ & $1.8 \mathrm{rad} / \mathrm{s}$ \\
Gain $k$ & $5 \times 10^{-2}$ & $6 \times 10^{-4}$ \\
\hline
\end{tabular}


(a)

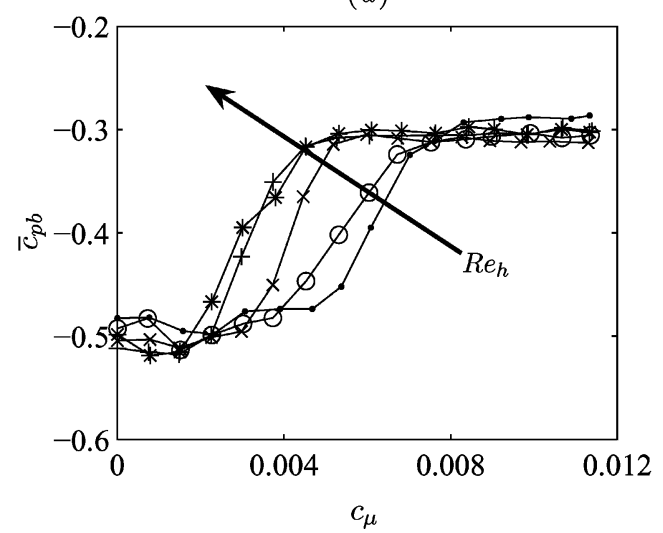

(b)
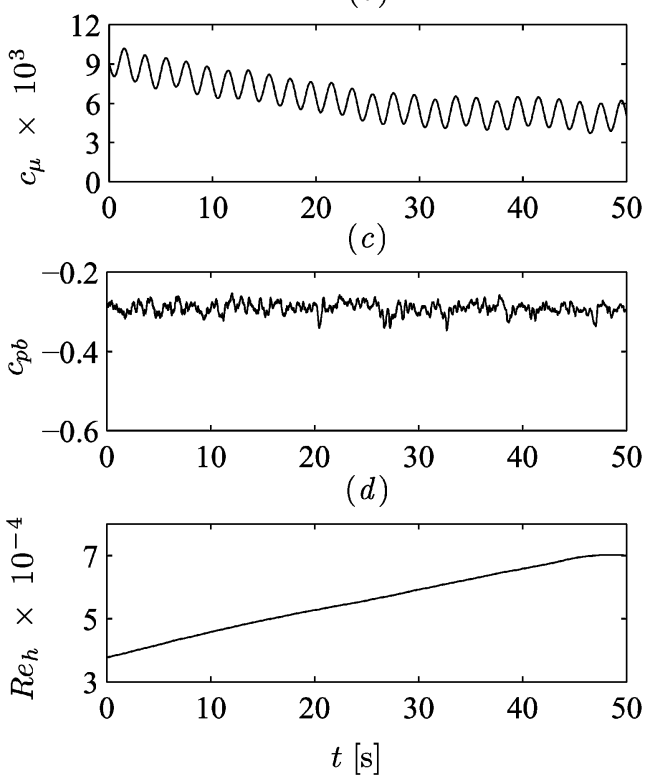

Fig. 8 Slope-seeking feedback in an experiment with an increasing Reynolds numbers (b-d). Steady state maps $\bar{c}_{p b}=f\left(c_{\mu}\right)$ are displayed (a) for various Reynolds numbers $R_{h}=23000$ $(\odot), 35000(\bigcirc), 46000(\times), 58000(+)$, and $70000(*)$. These maps are used only to indicate the success of control. They are not required for the controller. The Strouhal number of the in-phase forcing is $S t_{a}=0.15$

\subsubsection{Least squares estimation of the slope}

The experimental results with the slope-seeking controller extended by the least squares algorithm are shown in Fig. 9. All controller parameters $\left(u_{0}\right.$, $\left.\omega_{\text {sin }}, a, k, f_{\text {ref }}^{\prime}\right)$ are the same as before. For equation (10), $m=2$ and $n=2$ are chosen.

The controller starts again at $u_{0}=c_{\mu}=2 \times 10^{-3}$ with stepwise variation of the control input (Fig. 9(a)). The estimated gradient $\hat{f}^{\prime}$ is given in Fig. 9(d). Corresponding to the steady state map in Fig. 7 , the gradient increases for $0 \mathrm{~s} \leqslant t \leqslant 4 \mathrm{~s}$. At the optimal operating point the algorithm estimates a small gradient, as can be seen in Fig. 9(d), which is in good agreement with the identified steady state map. The extended controller achieves the optimum in approximately $4 \mathrm{~s}$, which is 20 per cent faster than the standard slope-seeking controller (see Fig. 7).

\subsubsection{Gradient estimation with a Kalman filter}

The experimental results with the slope-seeking controller extended by the Kalman filter are shown in Fig. 10. The time delay is set to $n \Delta t=2 \pi /\left(3 \omega_{\sin }\right)$ $\mathrm{s}=660 \Delta t$, with the sampling time $\Delta t=0.001 \mathrm{~s}$. The used parameters for the extended Kalman filter are given in the Appendix. A three times faster response is observed compared to the classical approach due to the fast estimation of the local gradient $\hat{f}^{\prime}$ (see
Fig. 7(d)). A further increase in performance for the extended slope-seeking controller cannot be achieved, since the time constant of the process is limiting the dynamics of the closed-loop.

\subsection{Active drag reduction for a three-dimensional car model (Ahmed body)}

A sketch of the Ahmed body configuration is shown in Fig. 11. The flow field in the wake is highly threedimensional, unsteady, and it strongly depends on the rear slant angle $\Phi_{s}$, as shown in references [41] and [42]. Longitudinal vortices occur at slant angles up to $30^{\circ}$, leading to a dramatic increase in pressure drag.

The experimental investigations are conducted in a wind tunnel with a closed test section. The shape of the generic car model investigated is based on the original geometry of the Ahmed body in reference [41] with a slant angle of $\Phi_{\mathrm{s}}=25^{\circ}$. In the experiments all dimensions (length $l=261 \mathrm{~mm}$, height $h=72 \mathrm{~mm}$, and width $w=97 \mathrm{~mm}$; see Fig. 11) are scaled to a quarter of the original sizes. The Reynolds number based on the free stream velocity $u_{0}$ and the model height $h$ is $R e_{h}=96000$.

The positions of actuators and sensors are shown in the sketch (Fig. 11). For steady blowing, cavities are integrated into the slant corners of the Ahmed 
(a)
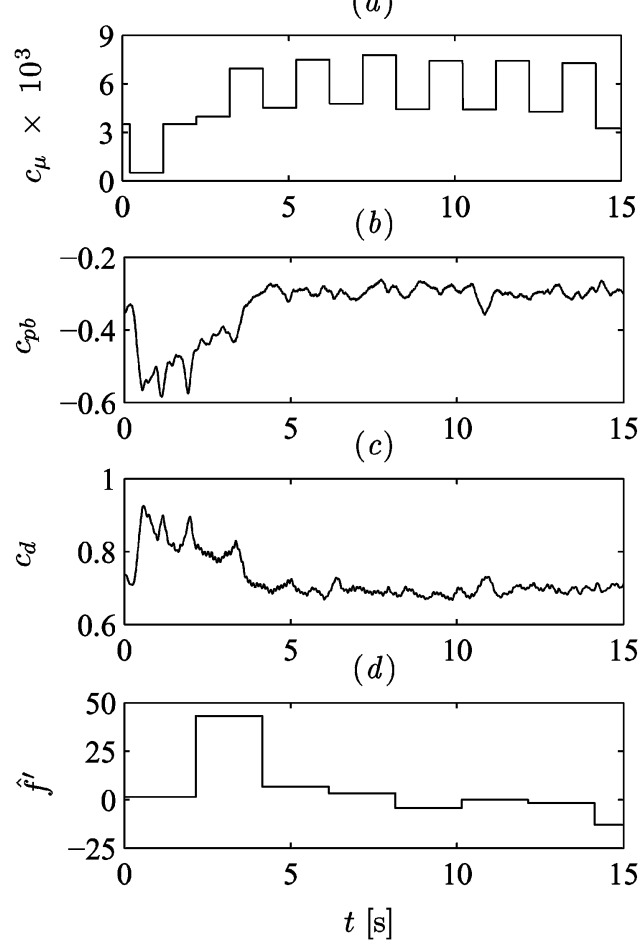

Fig. 9 Results obtained with a least squares extended slope-seeking controller. Experiments on the D-shaped body configuration with $R e_{h}=$ 46000 . For the estimation of the local gradient the controller changes the input stepwise (a). The maximum pressure can be achieved faster (b) compared to the standard slope-seeking controller, which gives rise to a reduction of the drag of approximately 15 per cent (c). In (d) the time series of the estimated gradient is given

body. The steady blowing intensity is adjusted by a magnetic valve (FESTO MPPES-3-1/4-6-010) within the range $0 \leqslant c_{\mu} \leqslant 7 \times 10^{-3}$, where the dimensionless momentum coefficient is now defined as

$$
c_{\mu}=\frac{A_{\mathrm{a}} q_{\mathrm{a}}^{2}}{h w u_{0}^{2}}
$$

with $A_{\mathrm{a}}=36 \mathrm{~mm}^{2}$ being the active actuator area. The synthetic jet is directed normal to the mean flow direction to avoid thrust generation due to the forcing.

A force balance in the low-speed wind tunnel, which consists of a sensitive strain gauge sensor (HBM KD 45) located below the test section, is used in order to measure the effect of the applied flow control to the total drag of the Ahmed body. The definition in equation (20) is applied for the determination of the dimensionless drag coefficient (a)
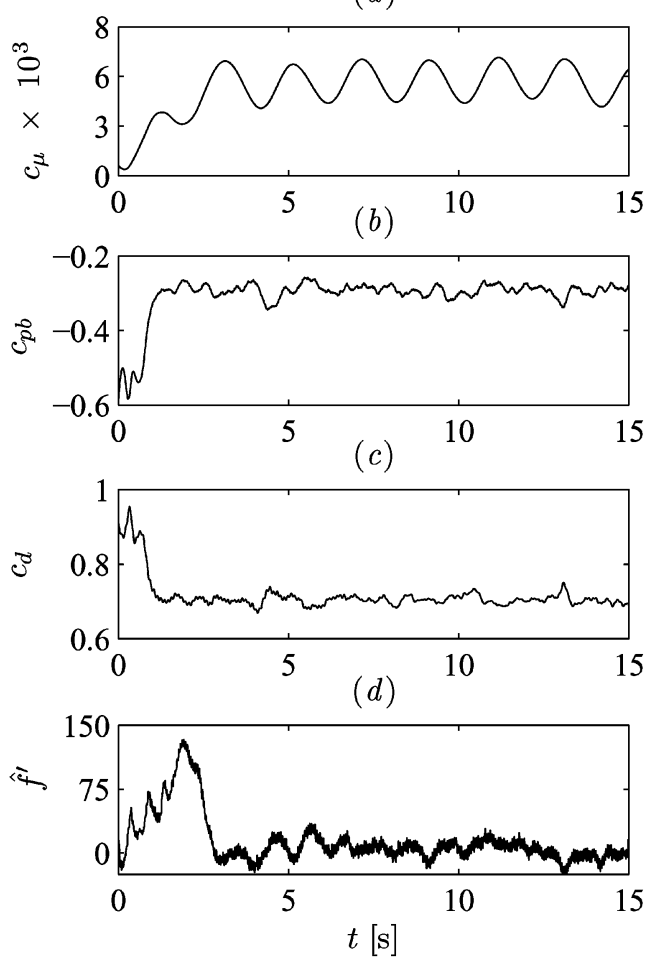

Fig. 10 A fast extended Kalman filter algorithm is used to estimate the current slope (d) of the steady state map. Thus the slope-seeking controller can achieve the optimal momentum coefficient faster (a), which leads to an increase in base pressure (b) and a reduction of the drag coefficient (c) of the D-shaped body. Harmonic in-phase actuation with $S t_{a}=0.15$ at $R e_{h}=46000$ is applied

$c_{d}$. Three pressure sensors (PascaLine PCLA02X5D1) are used for the measurement of the spatially averaged base pressure $c_{p b}$. The implementation of the controllers and the data acquisition are realized by a modular hardware for rapid control prototyping (dSPACE PPC1005). The sampling frequency is $1000 \mathrm{~Hz}$.

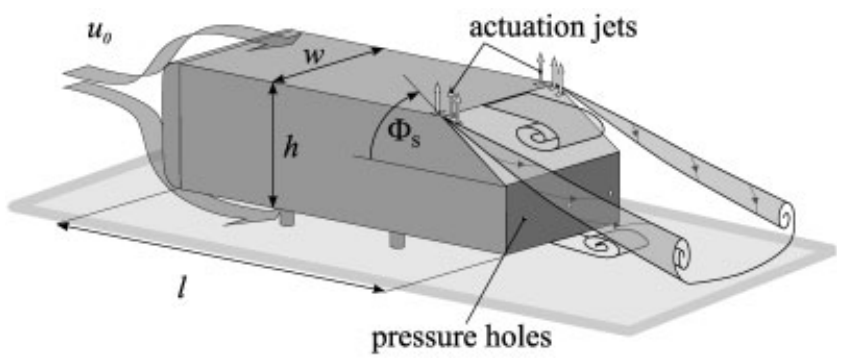

Fig. 11 Sketch of the Ahmed body with illustrated vortex structures behind the rear end and actuation position for steady blowing at the corners 
$(a)$

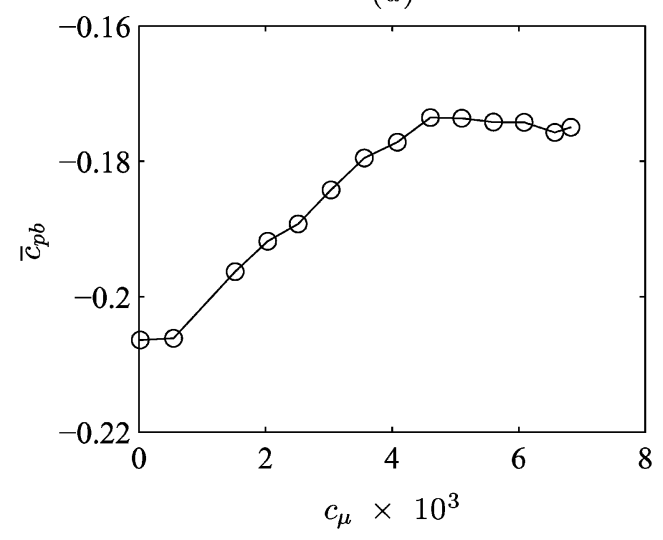

(b)
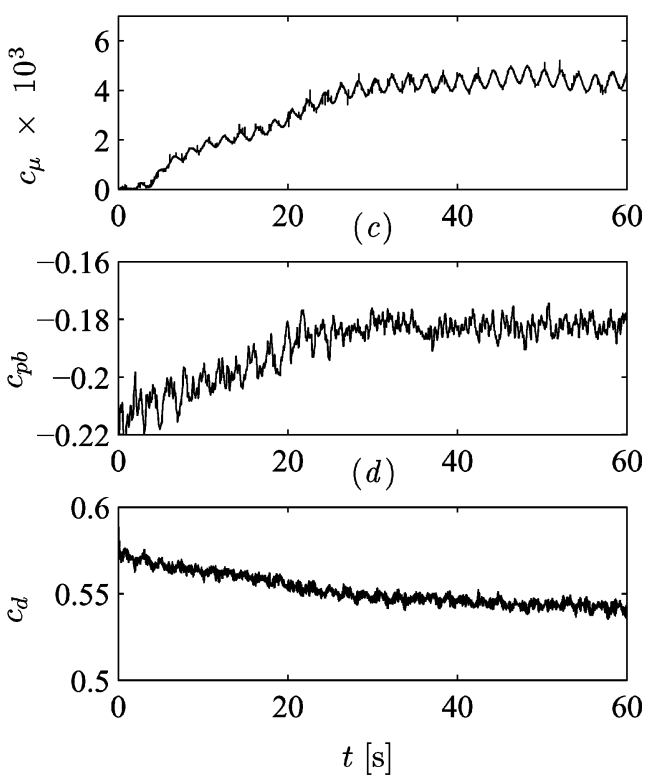

Fig. 12 Steady state map $\bar{c}_{p b}=f\left(c_{\mu}\right)$ obtained from open-loop experiments (a) and experimental results of the slope-seeking feedback for optimal drag reduction of the Ahmed body (b-d). The controller adapts the actuation amplitude (b) until the maximum base pressure (c) is reached and a maximum drag reduction of approximately 5 per cent is achieved. All experiments are conducted with steady blowing. Reynolds number is constant at $R e_{h}=96000$

\subsubsection{Classical slope-seeking control}

The numerical and experimental results in reference [32] show that through steady blowing near the slant corners the longitudinal vortices are weakened for the Ahmed body with a slant angle $\Phi_{\mathrm{s}}=25^{\circ}$. A suitable blowing intensity increases the base pressure and a small decrease of the aerodynamic drag is observed. Furthermore, a very good correlation of time-averaged drag and base pressure is observed. Therefore, the base pressure $c_{p b}$ is used as an output variable in this application.

Figure 12(a) shows the time-averaged base pressure as a function of the momentum coefficient for $R e_{h}=96000$, which is obtained from parameter studies. This steady state map $\bar{c}_{p b}=f\left(c_{\mu}\right)$ indicates an approximately linear slope in the range $0 \leqslant c_{\mu} \leqslant 4.5 \times 10^{-3}$. For a momentum coefficient greater than $4.5 \times 10^{-3}$ the base pressure $\bar{c}_{p b}$ shows a saturation that gives rise to the plateau-type steady state map.

The focus of the slope-seeking control is the adaptation of $c_{\mu}$ for maximum base pressure at minimum control input. Hence, the momentum coefficient is chosen as the control input, i.e. $u(t)=c_{\mu}(t)$. A standard slope-seeking controller is implemented in the experiments as described in section 2.2. The selected parameters of the controller are given in Table 1.
The experimental results with the controller at $R e_{h}=96000$ are shown in Figs 12(b) to (d). The control starts at $t=0 \mathrm{~s}$ with $u_{0}=c_{\mu}=0$ (Fig. 12(b)). The controller increases the input continuously, which yields an increase in base pressure (Fig. 12(c)). At $t=30 \mathrm{~s}$, the optimal momentum coefficient $c_{\mu} \approx 4.5 \times 10^{-3}$ is achieved and the base pressure is increased by approximately 15 per cent, corresponding to the steady state map in Fig. 12(a). A drag reduction of approximately 5 per cent can be observed in the time series of $c_{d}(t)$ in Fig. 12(d).

\subsubsection{Adaptation of the search radius}

Figure 13 shows the experimental results for the adaptation of the search radius for a slightly increased Reynolds number, $R e_{h}=106000$. The perturbation of the input signal starts with an amplitude of $a=3.6 \times 10^{-4}$ (see Fig. 13(c)). For $0 \mathrm{~s} \leqslant t \leqslant 90 \mathrm{~s}$, the controller increases the input $c_{\mu}(t)$ due to a large gradient in the steady state map (see Fig. 12(a)). Thus, the amplitude increases from $a=3.6 \times 10^{-4}$ to $a=4 \times 10^{-4}$. Around $t=80 \mathrm{~s}$ the controller has reached the reference slope. The gradient of the steady state map is small, and thus the controller decreases the perturbation amplitude continuously to approximately $2 \times 10^{-4}$. 
(a)

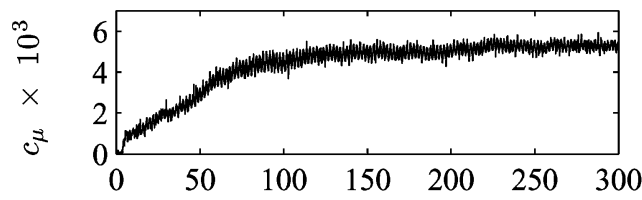

(b)

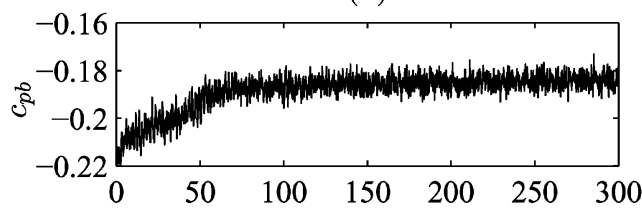

(c)

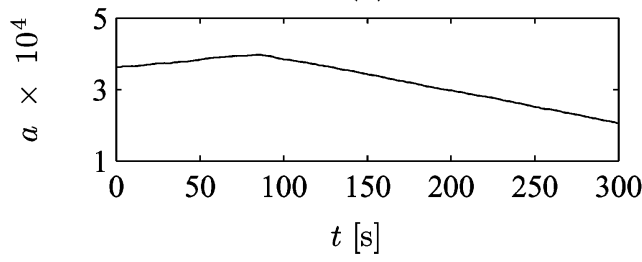

Fig. 13 Optimal drag reduction of the Ahmed body by the extended slope-seeking controller with adaptation of the perturbation amplitude. The controller decreases the perturbation amplitude after the optimal operating point has been achieved (c). Experimental results with $R e_{h}=106000$

The parameters of the controller (gain $k$, reference slope $f_{\text {ref }}^{\prime}$, perturbation frequency $\omega_{\text {sin }}$ ) are the same as these chosen in section 3.2. The gain $k_{a}$ is set to $k_{a}=1.0$ and $k_{r}$ has the value $k_{r}=5.0$.

\section{CONCLUSION AND OUTLOOK}

This paper illustrates the successful application of closed-loop control in several flow configurations. The flow over a D-shaped bluff body and a three-dimensional car model are controlled by the use of slope-seeking and extensions/modifications of the slope-seeking control algorithm. The basic slope-seeking controller reduces the drag of the Dshaped bluff body by 15 per cent and that of the three-dimensional car model by 5 per cent.

Starting from the basic slope-seeking control scheme, extensions of the slope-seeking algorithm are implemented and tested successfully in experiments. To tackle the waste of energy during the sinusoidal perturbation of the control input while operating in the optimal point, an adaptation of the search radius is proposed. Tests on the Ahmed body show that after reaching the optimal point the extension reduces the perturbation amplitude by one half, thus leading to a more energy-efficient implementation of the slope-seeking controller. The two presented modifications of the slope-seeking controller are introduced in order to obtain a faster estimation of the gradient. Firstly, a least squares algorithm is tested on the D-shaped bluff body. This extension leads to an approximately 20 per cent reduction of the time needed to reach the optimal point. Another approach to estimate the gradient is based on an extended Kalman filter. The time for reaching the optimal point is reduced here by approximately 66 per cent. Despite this acceleration the closed-loop is still slow compared to a more classical control loop. Therefore, other approaches mentioned in section 1 will remain important when a control in the time scale of the process dynamics is necessary. One obvious approach is to use extremum-seeking control for a slow adaptation of a reference value, which is then exploited in an inner and faster control loop. In future investigations, such ideas will be extended to more complex, practical flow configurations, e.g. a complete aircraft model in a large-scale wind-tunnel, a compressor, or a more realistic car model.

\section{ACKNOWLEDGEMENT}

Part of this work is funded by the 'Deutsche Forschungsgemeinschaft' (DFG) as part of the Collaborative Research Centre (Sfb 557) 'Control of complex turbulent shear flows' at Technische Universität Berlin.

\section{REFERENCES}

1 Bearman, P. W. Investigation of the flow behind a two-dimensional model with a blunt trailing edge and fitted with splitter plates. J. Fluid Mechanics, 1965, 21, 241-255.

2 Tanner, M. A method of reducing the base drag of wings with blunt trailing edges. Aeronaut. Q., 1972, 23, 15-23.

3 Tombazis, N. and Bearman, P. W. A study of threedimensional aspects of vortex shedding from a bluff body with a mild geometric disturbance. J. Fluid Mechanics, 1997, 330, 85-112.

4 Park, H., Lee, D., Jeon, W.-P., Hahn, S., Kim, J., Kim, J., Choi, J., and Choi, H. Drag reduction in flow over a two-dimensional bluff body with a blunt trailing edge using a new passive device. J. Fluid Mechanics, 2006, 563, 389-414.

5 Gad-el-Hak, M., Pollard, A., and Bonnet, J.-P. Flow control - fundamentals and practices, 1998 (Springer, Berlin, Heidelberg).

6 Greenblatt, D. and Wygnanski, I. J. The control of flow separation by periodic excitation. Prog. Aerospace Sci., 2000, 36, 487-545. 
7 Choi, H., Moin, P., and Kim, J. Active turbulence control for drag reduction in wall-bounded flows. J. Fluid Mechanics, 1994, 262, 75-110.

8 Bewley, T. R. and Liu, S. Optimal and robust control and estimation of linear paths to transition. J. Fluid Mechanics, 1998, 365, 305-349.

9 Coller, B. D., Noack, B. R., Narayanan, S., Banaszuk, A., and Khibnik, A. I. Reduced-basis model of active separation control in a planar diffusor flow. AIAA paper 2000-2562, 2000.

10 Wee, D., Park, S., Miake-Lye, R., Annaswamy, A. M., and Ghoniem, A. F. Reduced order modeling of reacting shear layers. AIAA paper 2002-0478, 2002.

11 Gerhard, J., Pastoor, M., King, R., Noack, B. R., Dillmann, A., Morzynski, M., and Tadmor, G. Model-based control of vortex shedding using lowdimensional Galerkin models. AIAA paper 20034262, 2003.

12 Pastoor, M., King, R., Noack, B. R., Dillmann, A., and Tadmor, G. Model-based coherent-stucture control of turbulent shear flows using low-dimensional vortex models. AIAA paper 2003-4261, 2003.

13 Rowley, C. W. and Williams, D. R. Control of forced and self-sustained oscillations in the flow past a cavity. AIAA paper 2003-0008, 2003.

14 Siegel, S., Cohen, K., and McLaughlin, T. Experimental variable gain feedback control of a cylinder wake. AIAA paper 2004-2611, 2004.

15 Allan, B. G., Juang, J.-N., Raney, D. L., Seifert, A., Pack, L. G., and Brown, D. E. Closed-loop separation control using oscillatory flow excitation. ICASE report 2000-32, 2000

16 King, R., Becker, R., Garwon, M., and Henning, L. Robust and adaptive closed-loop control of separated shear flows. AIAA paper 2004-2519, 2004.

17 Becker, R. and King, R. Comparison of a robust and a flatness based control for a separated shear flow. In 16th International Federation of Automatic Control World Congress (IFAC 2005), Prague, Czech Republic, July 2005.

18 Henning, L. and King, R. Robust multivariable closed-loop control of a turbulent backward-facing step flow. Am. Inst. Aeronaut. Astronaut. J. of Aircraft, 2007, 40(1), 201-208.

19 Garwon, M. and King, R. A multivariable adaptive control strategy to regulate the separated flow behind a backward-facing step. In Proceedings of the 16th IFAC World Congress, Prague, Czech Republic, July 2005.

20 King, R., Becker, R., Feuerbach, G., Henning, L., Petz, R., Nitsche, W., Lemke, O., and Neise, W. Adaptive flow control using slope seeking. In Proceedings of the 14th IEEE Mediterranean Conference on Control automation, Ancona, Italy, 2006.

21 Garwon, M., Urzynicok, F., Darmadi, L. H., Bärwolff, G., and King, R. Adaptive control of separated flows. In Proceedings of the European Control Conference 2003 (ECC03), Cambridge, 2003.
22 Moeck, J. P., Bothien, M. R., Paschereit, C. O., Gelbert, G., and King, R. Two-parameter extremum seeking for control of thermoacoustic instabilities and characterization of linear growth. AIAA paper 2007-1416, 2007.

23 Krstic, M. and Wang, H.-H. Stability of extremum seeking feedback for general nonlinear dynamic systems. Automatica, 2000, 36, 595-601.

24 Ariyur, K. and Krstic, M. Real-time optimization by extremum-seeking control, 2003 (John Wiley \& Sons, Inc., Hoboken).

25 Kim, J., Hahn, S., Kim, J., Lee, D., Choi, J., Jeon, W.-P., and Choi, H. Active control of turbulent flow over a model vehicle for drag reduction. J. Turbulence, 2004, 5(019)

26 Bearman, P. W. The effect of base bleed on the flow behind a two-dimensional model with a blunt trailing edge. Aeronaut. Q., 1967, 18, 207-224.

27 Grosche, F. R. and Meier, G. E. A. Research at the DLR Göttingen on bluff body aerodynamics. J. of Wind Engng and Ind. Applic., 2001, 89, 1201-1218.

28 King, R., Seibold, S., Lehmann, O., Noack, B. R., Morzynski, M., and Tadmor, G. Nonlinear flow control based on a low dimensional model of fluid flow. In T. Meurer et al., editor, Lecture Notes in Control and Information Sciences (Eds Meurer et al.), vol. 322, 2005, pp. 369-386 (Springer, Berlin, Heidelberg).

29 Beaudoin, J. F., Cadot, O., Aider, J.-L., and Wesfreid, J.-E. Drag reduction of a bluff body using adaptive control methods. Physics of Fluids, 2006, 18(085107), 1-10.

30 Henning, L. and King, R. Drag reduction by closedloop control of a separated flow over a bluff body with a blunt trailing edge. In 44th Institute of Electrical and Electronics Engineers (IEEE) Conference on Decision and control and European Control Conference (ECC) 2005, December 2005.

31 Brunn, A. and Nitsche, W. Active control of turbulent separated flows by means of large scale vortex excitation. In Engineering turbulence modelling and experiments (Eds W Rodi and M Mulas), 2005 no. 6, pp. 555-564 (Elsevier Science Ltd, Oxford).

32 Brunn, A., Wassen, E., Sperber, D., Nitsche, W., and Thiele, F. Active drag control for a generic car model. In Active flow control (Ed R King), vol. 95 of Notes on numerical fluid mechanics and multidisciplinary design (NNFM), 2007, pp. 247-259 (Springer, Berlin, Heidelberg).

33 Schulz, J., Garwon, M., Satriadarma, B., King, R., Möser, M., and Neise, W. Adaptive and robust control for the reduction of tonal noise components of axial turbomachinery with flow control. In Proceedings of DAGA 2004, Strasbourg, France, 2004.

34 Henning, L., Pastoor, M., King, R., Noack, B. R., and Tadmor, G. Feedback control applied to bluff body wake. In Active flow control (Ed R King), 
vol. 95 of Notes on numerical fluid mechanics and multidisciplinary design (NNFM), 2007, pp. 369-391 (Springer, Berlin, Heidelberg).

35 Morosanov, I. S. Method of extremum control. Automn Remote Control, 1957, 18, 1077-1092.

36 Soederstroem, T. and Stoica, P. System identification, 1989 (Prentice Hall, Englewood Cliffs, New Jersey).

37 Ljung, L. System identification - theory for the user, 1999 (Prentice Hall PTR, Englewood Cliffs, New Jersey).

38 Gelb, A. Applied optimal estimation, 1986 (The MIT Press, Cambridge, Massachusetts).

39 Huerre, P. and Monkewitz, P. A. Local and global instabilities in spatially developing flows. Annual Rev. Fluid Mechanics, 1990, 22, 473-537.

40 Mercker, E. Eine Blockierungskorrektur für aerodynamische Messungen in offenen und geschlossenen Unterschallwindkanälen, $\mathrm{PhD}$ thesis, Technische Universität Berlin, 1980.

41 Ahmed, S. R., Ramm, R., and Faltin, G. Some salient features of the time-averaged ground vehicle wake. SAE paper 840300, 1984.

42 Lienhart, H., Stoots, C., and Becker, S. Flow and turbulence structures in the wake of a simplied car model (Ahmed model). In Notes on numerical fluid mechanics, 2002, no. 77, pp. 323-330 (Vieweg, Braunschweig).

\section{APPENDIX}

\section{Parameters of the Kalman filter}

The following parameters are used for the design of the extended Kalman filter applied in section 3.1. The covariance matrix of the process noise is given as

$$
\mathbf{Q}_{k}=\left[\begin{array}{cc}
1 \times 10^{-2} & 0.0 \\
0.0 & 4 \times 10^{2}
\end{array}\right] \Delta t
$$

with the sampling time $\Delta t$. The covariance matrix of the measurement noise is set to

$$
\mathbf{R}_{k}=\left[\begin{array}{cc}
1 \times 10^{-2} & 0.0 \\
0.0 & 1 \times 10^{-2}
\end{array}\right]
$$

and for the initial covariance matrix of the estimation error

$$
\mathbf{P}_{0}=\left[\begin{array}{cc}
1 \times 10^{-2} & 0.0 \\
0.0 & 1 \times 10^{2}
\end{array}\right]
$$

is used. 